The National Bureau of Standards does not issue house plans, but has compiled the following list of sources from which house plan booklets, stock plans for houses, floor plans, or suggestions for small house design may be obtained. Architects everywhere, of course, design plans for individual needs and many retail lumber dealers maintain house plan service departments. House plans, particularly for farm houses, are also available from some state colleges. Floor plans, descriptions, and illustrations published by leading newspapers, architectural, building, and home magazines may be consulted in large libraries.

\section{FEDERAL AGENCIES}

U. S. Department of Agricul ture Bureau of Plant Industry, Soils, and Agricul tural Engineering (Farm Homes)

Bel tsville, Maryl and

Federal Housing Administration Vermont Ave. and $\mathrm{K}$ Street $\mathrm{N}$. $\mathrm{W}$. Washington 25, D. C.

Housing and Home Finance Agency $1626 \mathrm{~K}$ S treet N. W. Washington 25, D. C。

Tennessee Valley Authori ty Architectural Design Branch Design Division Knoxville, Tennessee

\section{TRADE ASSCCIATIONS}

Home Builders Association of Greater Cleveland Allerton Hotal

E. 13th Street \& Chester Ave. Cleveland 14, Ohio

Northeastern Retail Lumbermens Association

82 St. Paul Street

Rochester 4, New York

\section{TRADF ASSOCIAIIONS.--Cont.}

Southem Pine Association

520 Canal Building

New Orl eans 4, Io uisiana

Structural Clay Products Institute $1756 \mathrm{~K}$ Street $\mathbb{N}$. $\pi$. Wasnington 6, D. C.

Structural Clay Products Institute, Inc.

Ohio Region

Renkert Buil ding

Canton 2, Ohio

\section{PERIODICAIS}

American Lumbernan and Building

Products Merchandiser

139 N. Clark Street

Chicago 2, Illinois

Better Homes and Gardens

Home planning Service

Des Moines 3, Iowa

Parents' Magazine

Expandable Eomes

(Designed especially for families with children)

52 Vanderbilt Avenue

New York 17, N. Y. 


\section{PERIODICALS.--Contd.}

Practical Builder

5 S. Wabash Avenue

Chicago 3, Illinois

Toman's Home Companion

(House building)

250 Park Avenue

New York 17, N. Y.

\section{CONPANIES}

Architects' Home Plan Institute Box 988

Minneapol is, Minne so ta

Brown-Blodgett, Inc.

407 Empire Bank Buil ding

St. Paul 1, Minnaso ta

Cleval and Publications

House Plan Dent. U

448 S. Hill Street

Los Angeles 13, California.

I. F. Garl inghouse Co., Inc.

115 - 8th Avenue, East

Topeka, Kansas

Griffen-Patterson Company

544 T. Colorado Boul evard

Glendal a 4, California

Home Book Eublishers

145 Second Street

San Francisco 5, California

Home Plan Book Co.

1596 Selby Avenue

st. Paul 4, Minnesota

Home of the Teak Plan Service

89 Elmgrove Avenue

Providence 6, Rhode Island

National Plan Co. of Oregon

405 Pearson-Fourth Avenue BIdg.

Portland 4, Oragon

National Plan Service, Inc.

$1315 \pi$. Congress Street

chicago 7, Illinois
COMPANIES. - - Contd.

N. . Plan Service

125 South 10th Street

Minneapolis 2, Minnesota

Wm. E. Padersen

210 Globe Building

St. Paul 1, Minnesota

Standard Homes Company

Colorado Buil ding

Washington 5, D. C.

Universal Plan Service

332 Builders Exchange Building

320 S. T. Stari street

Portland 4, Oregon 